\title{
Successive Approximation of SFDEs with Finite Delay Driven by G-Brownian Motion
}

\author{
Litan Yan ${ }^{1,2}$ and Qinghua Zhang ${ }^{1}$ \\ ${ }^{1}$ Glorious Sun School of Business and Management, Donghua University, 1882 West Yan-an Rood, Shanghai 200051, China \\ ${ }^{2}$ Department of Mathematics, Donghua University, 2999 North Renmin Rood, Songjiang, Shanghai 201620, China
}

Correspondence should be addressed to Litan Yan; litanyan@dhu.edu.cn and Qinghua Zhang; zhangqhalpha@gmail.com

Received 11 November 2013; Accepted 5 December 2013

Academic Editor: Yaozhong $\mathrm{Hu}$

Copyright (c) 2013 L. Yan and Q. Zhang. This is an open access article distributed under the Creative Commons Attribution License, which permits unrestricted use, distribution, and reproduction in any medium, provided the original work is properly cited.

We consider the stochastic functional differential equations with finite delay driven by G-Brownian motion. Under the global Carathéodory conditions we prove the existence and uniqueness, and as an application, we price the European call option when the underlying asset's price follows such an equation.

\section{Introduction}

Motivated by various types of uncertainty and financial problems, Peng [1] has introduced a new notion of nonlinear expectation, the so-called $G$-expectation (see also Peng et al. [2-4]), which is associated with the following nonlinear heat equation:

$$
\begin{gathered}
\frac{\partial}{\partial t} u(t, x)=G(\Delta u), \quad(t, x) \in[0,+\infty) \times \mathbb{R}, \\
u(0, x)=\varphi(x),
\end{gathered}
$$

where $\Delta$ is Laplacian and the sublinear function $G$ is defined as

$$
G(\alpha)=\frac{1}{2}\left(\bar{\sigma}^{2} \alpha^{+}-\underline{\sigma}^{2} \alpha^{-}\right), \quad \alpha \in \mathbb{R}
$$

with two given constants $0<\underline{\sigma}<\bar{\sigma}$. Together with the notion of $G$-expectations Peng also introduced the related $G$-normal distribution, the $G$-Brownian motion, and related stochastic calculus under $G$-expectation, and moreover an Itô's formula for the G-Brownian motion was established. $G$-Brownian motion has a very rich and interesting new structure which nontrivially generalizes the classical one. Briefly speaking, a $G$-Brownian motion $B$ is a continuous process with independent stationary increments $B_{t+s}-$ $B_{t}$ being $G$-normally distributed under a given sublinear expectation $\widehat{\mathbb{E}}$. A very interesting new phenomenon of $G^{-}$ Brownian motion $B$ is that its quadratic process $\langle B\rangle$ is a continuous process with independent and stationary increments, but not a deterministic process.

On the other hand, over the past decades, thanks for the contribution of Black and Scholes [5] and Merton [6] in the formulation of Black-Scholes model, the trading of derivatives has become an important area in the world of finance, and the Black-Scholes formula has been one of the most important consequences of the study of continuous time models in finance. Although Black-Scholes model is a benchmark of mathematical finance, the significant weakness of it cannot be ignored since empirical evidence shows that volatility actually depends on time in a way that is not predictable and many empirical studies have outlined that the volatility of underlying asset is highly unlikely to be constant. This is sometimes pointed out as the reason for inaccurate predictions made by the Black and Scholes formula. Therefore, a number of alternative methods have been studied for the underlying asset model. In this paper, we consider the effect of the past in the determination of the fair price of a call option under a sublinear framework. In particular, we assume that the stock price satisfies a stochastic functional differential equation (SFDE) with fixed or variable delay driven by a $G$-Brownian motion. We consider call options that can be exercised only at the maturity date, namely, European call options. Let $\{B(t), t \geq 0\}$ be 
the $G$-Brownian motion with the quadratic variation process $\{\langle B\rangle(t), t \geq 0\}$. We consider SFDE with finite delay of the form

$$
\begin{array}{r}
d X(t)=b\left(t, X_{t}\right) d t+h\left(t, X_{t}\right) d\langle B\rangle(t)+\sigma\left(t, X_{t}\right) d B(t), \\
t \in\left[t_{0}, T\right],
\end{array}
$$

where (i) $X_{t}=\{X(t+\theta)$; $-\tau \leq \theta \leq 0\}$ and $t \in\left[t_{0}, T\right]$ is a $C([-\tau, 0] ; \mathbb{R})$-valued stochastic process; (ii) $b, h$, and $\sigma$ are three given functions satisfying some satiable conditions; (iii) $\tau>0$ and $0 \leq t_{0}<\infty$ are two given constants.

As an application we price the European call option when the underlying asset's price follows a special SFDE. In the case of classical Brownian motion, this is first studied by Arriojas et al. [7].

The note is organized as follows. In Section 2, we present some preliminaries for sublinear expectation and $G$ Brownian motion $B$. In Section 3, we express and prove our main result. In Section 4, we give an application to price the European call option when the underlying asset price follows a special SFDE driven by a $G$-Brownian motion.

\section{Preliminaries}

In this section, we briefly recall some basic notations and results for $G$-Brownian motion under $G$-framework. For more aspects on these material we refer to Peng [4], Denis et al. [8], and $\mathrm{Hu}$ and Peng [9]. More works for G-Brownian motion can be found in $\mathrm{Hu}$ and $\mathrm{Li}$ [10], Lin [11], Peng et al. [12], Song [13], Xu et al. [14], Yan et al. [15, 16], and the references therein.

2.1. Sublinear Expectation Space. Let $\Omega \neq \emptyset$ be a given set and let $\mathscr{H}$ be a linear space of real-valued functions defined on $\Omega$ such that $1 \in \mathscr{H}$ and $|X| \in \mathscr{H}$ for all $X \in \mathscr{H}$. It is important to note that we can suppose that

$$
\varphi\left(X_{1}, \ldots, X_{d}\right) \in \mathscr{H}
$$

if $X_{i} \in \mathscr{H}, i=1, \ldots, d$, for all $\varphi \in C_{b \text {, Lip }}\left(\mathbb{R}^{d}\right)$, where $C_{b, \text { Lip }}\left(\mathbb{R}^{d}\right)$ denotes the space of all bounded and Lipschitz functions on $\mathbb{R}^{d}$.

Definition 1. A sublinear expectation $\widehat{\mathbb{E}}$ on $\mathscr{H}$ is a functional with the following properties: for all $X, Y \in \mathscr{H}$, we have

(i) monotonicity: if $X \geq Y$, then $\widehat{\mathbb{E}}[X] \geq \widehat{\mathbb{E}}[Y]$;

(ii) constant preserving: $\widehat{\mathbb{E}}[c]=c$, for all $c \in \mathbb{R}$;

(iii) subadditivity: $\widehat{\mathbb{E}}[X]-\widehat{\mathbb{E}}[Y] \leq \widehat{\mathbb{E}}[X-Y]$;

(iv) positive homogeneity: $\widehat{\mathbb{E}}[\lambda X]=\lambda \widehat{\mathbb{E}}[X]$, for all $\lambda \geq 0$.

The triple $(\Omega, \mathscr{H}, \widehat{\mathbb{E}})$ is called a sublinear expectation space, and $\mathscr{H}$ is considered as the space of random variables on $\Omega$.
In this paper we throughout let $\Omega=C_{0}\left(\mathbb{R}^{+}\right)$be the space of all real-valued continuous functions on $[0, \infty)$ with initial value 0 , equipped with the distance

$$
\begin{array}{r}
\rho\left(\omega^{1}, \omega^{2}\right)=\sum_{i=1}^{\infty} 2^{-i}\left[\left(\max _{t \in[0, i]}\left|\omega_{t}^{1}-\omega_{t}^{2}\right|\right) \wedge 1\right], \\
\omega^{1}, \omega^{2} \in \Omega .
\end{array}
$$

We denote by $\mathscr{B}(\Omega)$ the Borel-algebra on $\Omega$. We also denote, for each $t \in[0, \infty)$,

$$
\Omega_{t}=\left\{\omega_{\cdot \wedge t}, \omega \in \Omega\right\}
$$

and $\mathscr{F}_{t}=\mathscr{B}\left(\Omega_{t}\right)$, where $x \wedge y=\min \{x, y\}$. Let $\mathbb{L}_{G}^{p}(\Omega)$ be the closure of $\mathscr{H}$ with respect to the norm

$$
\|X\|_{p}=\widehat{\mathbb{E}}\left[|X|^{p}\right]^{1 / p}
$$

with $p \in[1, \infty)$. Clearly, the space $\mathbb{L}_{G}^{p}(\Omega)$ is a Banach space and the space $C_{b}(\Omega)$ of bounded continuous functions on $\Omega$ is a subset of $\mathbb{L}_{G}^{p}(\Omega)$, and moreover, there exists a weakly compact family $\mathscr{P}$ of probability measures on $(\Omega, \mathscr{B}(\Omega))$ such that

$$
\widehat{\mathbb{E}}=\sup _{P \in \mathscr{P}} E_{P} .
$$

So we can introduce the Choquet capacity $\widehat{C}$ by

$$
\widehat{C}(A)=\sup _{P \in \mathscr{P}} P(A), \quad A \in \mathscr{B}(\Omega) \text {. }
$$

Definition 2. A set $A \subset \Omega$ is called polar if $\widehat{C}(A)=0$. A property is said to hold "quasi sure" (q.s.) if it holds outside a polar set.

The above family of probability measures $P$ allows characterizing the space $\mathbb{L}_{G}^{p}(\Omega)$ as follows:

$\mathbb{L}_{G}^{p}(\Omega)=\{X: \mathscr{B}(\Omega)=$ measurable real-valued functions on $\Omega$ such that $\sup _{P \in \mathscr{P}} E_{P}\left[|X|^{p}\right]<\infty$, and is $\widehat{C}$-quasi surely continuous $\}$.

Lemma 3 (Denis et al. [8]). Let $1 \leq p<\infty$. Consider the sets $\mathbb{L}_{G}^{p}(\Omega)$ and $\mathbb{L}^{p}=\mathscr{L}^{p} / \mathcal{N}$, where

$$
\begin{gathered}
\mathscr{L}^{p}=\{X: \mathscr{B}(\Omega)=\text { measurable real-valued functions } \\
\text { on } \left.\Omega \text { such that } \widehat{\mathbb{E}}\left(|X|^{p}\right)=\sup _{P \in \mathscr{P}} E_{P}\left[|X|^{p}\right]<\infty\right\}, \\
\mathscr{N}=\{X: \mathscr{B}(\Omega)=\text { measurable real-valued functions } \\
\text { on } \Omega \text { such that } X=0 \text { q.s }\} .
\end{gathered}
$$

Then

(i) $\mathbb{L}^{p}$ is a Banach space with respect to the norm $\|\cdot\|_{p}$;

(ii) $\mathbb{\square}_{G}^{p}$ is the completion of $C_{b}(\Omega)$ with respect to the norm $\|\cdot\|_{p}$. 
Lemma 4 (Li and Peng [17]). For a given $p \in(0,+\infty]$, If the sequence $\left\{X_{n}\right\} \subset \mathbb{L}^{p}$ converges to $X$ in $\mathbb{L}^{p}$, then there exists a subsequence $\left\{X_{n_{k}}\right\}$ such that $X_{n_{k}}$ converges to $X$ quasi surely.

Lemma 5 (see [18]). Let $\rho: \mathbb{R} \rightarrow \mathbb{R}$ be an increasing and concave function. Then the inequality

$$
\widehat{\mathbb{E}}[\rho(X)] \leq \rho(\widehat{\mathbb{E}}[X])
$$

holds for all $\mathscr{B}(\Omega)$-measurable real-valued functions on $\Omega$.

For $X=\left(X_{1}, \ldots, X_{n}\right) \in \mathscr{H}^{n}$, the functional $F_{X}$ defined by

$$
F_{X}[\varphi]:=\widehat{\mathbb{E}}[\varphi(X)], \quad \varphi \in C_{b, \operatorname{Lip}}\left(\mathbb{R}^{n}\right)
$$

is called the distribution of $X$ under $\widehat{\mathbb{E}}$. In a sublinear expectation space $(\Omega, \mathscr{H}, \widehat{\mathbb{E}})$, a random vector $Y=\left(Y_{1}, \ldots, Y_{n}\right)$, $Y_{i} \in \mathscr{H}$, is said to be independent under $\widehat{\mathbb{E}}$ from another random vector $X=\left(X_{1}, \ldots, X_{m}\right), X_{i} \in \mathscr{H}$, if for each test function $\varphi \in C_{b, \text { Lip }}\left(\mathbb{R}^{m+n}\right)$ we have

$$
\widehat{\mathbb{E}}[\varphi(X, Y)]=\widehat{\mathbb{E}}\left[\widehat{\mathbb{E}}[\varphi(x, Y)]_{x=X}\right] .
$$

2.2. G-Brownian Motion. Let $\underline{\sigma}, \bar{\sigma}$ be two real numbers with $0 \leq \sigma \leq \bar{\sigma}$.

A random variable $\xi$ in a sublinear expectation space $(\Omega, \mathscr{H}, \widehat{\mathbb{E}})$ is called $G$-normal distributed, denoted by $\xi \sim$ $N\left(0,\left[\underline{\sigma}^{2}, \bar{\sigma}^{2}\right]\right)$, if, for each $\varphi \in C_{b \text {,Lip }}(\mathbb{R})$, the function defined by

$$
u(t, x):=\widehat{\mathbb{E}}[\varphi(x+\sqrt{t} \xi)], \quad(t, x) \in[0, \infty) \times \mathbb{R}
$$

is the unique viscosity solution of the following nonlinear heat equation:

$$
\begin{gathered}
\frac{\partial}{\partial t} u(t, x)=G(\Delta u), \quad(t, x) \in[0,+\infty) \times \mathbb{R}, \\
u(0, x)=\varphi(x),
\end{gathered}
$$

where $\Delta$ is Laplacian and the sublinear function $G$ is defined as

$$
G(\alpha)=\frac{1}{2}\left(\bar{\sigma}^{2} \alpha^{+}-\underline{\sigma}^{2} \alpha^{-}\right), \quad \alpha \in \mathbb{R} .
$$

In particular, $E[\varphi(\xi)]=u(1,0)$ is the distribution of $\xi$.

Example 6 (Peng [1]). Let $\xi \sim N\left(0,\left[\underline{\sigma}^{2}, \bar{\sigma}^{2}\right]\right)$. We then have

$$
\widehat{\mathbb{E}}[\varphi(\xi)]=\frac{1}{\sqrt{2 \pi} \bar{\sigma}} \int_{\mathbb{R}} \varphi(x) e^{-\left(1 / 2 \bar{\sigma}^{2}\right) x^{2}} d x
$$

for all convex functions $\varphi$ and

$$
\widehat{\mathbb{E}}[\psi(\xi)]=\frac{1}{\sqrt{2 \pi} \underline{\sigma}} \int_{\mathbb{R}} \psi(x) e^{-\left(1 / 2 \underline{\sigma}^{2}\right) x^{2}} d x
$$

for all concave functions $\psi$.
Definition 7. A process $B=\{B(t), t \geq 0\} \subset \mathscr{H}$ in a sublinear expectation space $(\Omega, \mathscr{H}, \widehat{\mathbb{E}})$ is called a $G$-Brownian motion if the following properties are satisfied:

(i) $B(0)=0$;

(ii) for each $t, s \geq 0$, the increment $B(t+s)-B(t)$ is $N\left(0,\left[\sigma^{2} s, \bar{\sigma}^{2} s\right]\right)$-distributed and is independent of $\left(B\left(t_{1}\right), \ldots, B\left(t_{n}\right)\right)$, for all $n=0,1,2, \ldots$ and $0 \leq t_{1} \leq$ $t_{2} \leq \cdots \leq t_{n} \leq t$.

Recall that a process $X=\{X(t): 0 \leq t \leq T\}$ is called a $G$-martingale if, for each $0 \leq t \leq T$, we have $X(t) \in \mathbb{L}_{G}^{1}\left(\Omega_{t}\right)$ and

$$
\widehat{\mathbb{E}}\left[X(t) \mid \Omega_{s}\right]=X(s)
$$

for all $s \leq t$, and moreover, $X$ is called a symmetric $G$ martingale if both $X$ and $-X$ are $G$-martingales.

Remark 8. For simplicity throughout this paper we let $\underline{\sigma}=$ $\sigma_{0}>0$, and $\bar{\sigma}=1$.

2.3. Itô's Integral. We now recall the definition of Itô's integral and quadratic variation process of the G-Brownian motion. In Li and Peng [17], a generalized Itô integral and a generalized Itô formula with respect to the G-Brownian motion are discussed as follows. For arbitrarily fixed $p \geq 1$ and $T \in \mathbb{R}_{+}$, we first denote by $M_{G}^{p, 0}([0, T])$ the set of step processes

$$
\eta_{t}(\omega)=\sum_{j=1}^{N} \xi_{j}(\omega) I_{\left[t_{j-1}, t_{j}\right)}(t), \quad \xi_{j}(\omega) \in \mathbb{L}_{G}^{p}\left(\Omega_{t_{j}}\right)
$$

with $0=t_{0}<\cdots<t_{N}=T$. Moreover, we denote by $M_{G}^{p}([0, T])$ the completion of $M_{G}^{p, 0}([0, T])$ under the norm

$$
\|\eta\|_{M_{G}^{p}([0, T])}=\left(\widehat{\mathbb{E}}\left[\int_{0}^{T}|\eta(s)|^{p} d s\right]\right)^{1 / p} .
$$

Now we can define the stochastic integral $\int_{0}^{T} \eta(s) d B(s)$ for $\eta \epsilon$ $M_{G}^{p}([0, T])$.

Definition 9. For every $\eta \in M_{G}^{p, 0}([0, T])$ with the form (21), we define Itô's integral

$$
I(\eta)=\int_{0}^{t} \eta(s) d B(s):=\sum_{j=1}^{N} \xi_{j}\left(B\left(s_{j}\right)-B\left(s_{j-1}\right)\right) .
$$

One can show that $I(\eta) \in \mathbb{L}_{G}^{2}$ and the linear mapping (see Li and Peng [17])

$$
I: M_{G}^{2,0}([0, T]) \longrightarrow \mathbb{L}_{G}^{2}
$$

is continuous. Moreover, it can be continuously extended to

$$
I: M_{G}^{2}([0, T]) \longrightarrow \mathbb{L}_{G}^{2} .
$$


And, for all $\eta \in M_{G}^{2}([0, T])$, we have

$$
\begin{gathered}
\widehat{\mathbb{E}}\left(\int_{0}^{T} \eta(s) d B(s)\right)=0 \\
\widehat{\mathbb{E}}\left[\left(\int_{0}^{T} \eta(s) d B(s)\right)^{2}\right] \leq \bar{\sigma}^{2} \widehat{\mathbb{E}}\left[\int_{0}^{T} \eta^{2}(s) d s\right] .
\end{gathered}
$$

We now recall the definition of quadratic variation process of the $G$-Brownian motion $B$.

Definition 10 (quadratic variation). Let $\pi_{t}^{N}=\left\{0=t_{0}^{N}<t_{1}^{N}<\right.$ $\left.\cdots<t_{N-1}^{N}=t\right\}$ be a partition of $[0, t]$ for $t>0$, such that $\mu\left(\pi_{t}^{N}\right):=\max _{j}\left\{t_{j}-t_{j-1}\right\} \rightarrow 0$ as $N \rightarrow \infty$. The quadratic variation of $G$-Brownian motion $B$ can be defined as

$$
\begin{aligned}
\langle B\rangle(t) & =\lim _{\mu\left(\pi_{t}^{N}\right) \rightarrow 0} \sum_{k=0}^{N-1}\left(B\left(t_{k+1}^{N}\right)-B\left(t_{k}^{N}\right)\right)^{2} \\
& =B^{2}(t)-2 \int_{0}^{t} B(s) d B(s)
\end{aligned}
$$

in $\mathbb{L}_{G}^{2}$.

The function $t \mapsto\langle B\rangle(t)$ is continuous and increasing outside a polar set. We can define the integral

$$
\int_{0}^{T} \eta(t) d\langle B\rangle(t):=\sum_{j=1}^{N} \xi_{j}\left(\langle B\rangle\left(t_{j}\right)-\langle B\rangle\left(t_{j-1}\right)\right)
$$

as a map from $M_{G}^{1,0}([0, T])$ into $\mathbb{L}_{G}^{1}$, and the map is linear and continuous, so it can be extended continuously to $M_{G}^{1}([0, T])$.

Finally, we recall some important results in order to get our desired result. For detailed description of them, please read the related papers.

Theorem 11 (Itô's formula). Let $F \in C^{2,1}(\mathbb{R} \times[0, \infty))$. One then has

$$
\begin{aligned}
F(B(t), t)= & F(B(0), 0)+\int_{0}^{t} \frac{\partial}{\partial x} F(B(s), s) d B(s) \\
& +\int_{0}^{t} \frac{\partial}{\partial t} F(B(s), s) d s \\
& +\frac{1}{2} \int_{0}^{t} \frac{\partial^{2}}{\partial x^{2}} F(B(s), s) d\langle B\rangle(s)
\end{aligned}
$$

for all $t \geq 0$.

Lemma 12 (see [14]). If there exists an $\varepsilon_{0}>0$ such that

$$
\widehat{\mathbb{E}}\left[\exp \left\{\left(\frac{1}{2}+\varepsilon_{0}\right) \int_{0}^{T} H^{2}(s, \omega) d\langle B\rangle(s)\right\}\right]<\infty
$$

then the process

$$
B(t)-\int_{0}^{t} H(s, \omega) d\langle B\rangle(s), \quad 0 \leq t \leq T
$$

Lemma 13 (see [19]). Let $p \geq 2$ and $\eta=\{\eta(t), t \in[0, T]\} \in$ $M_{G}^{p}([0, T])$. Then

$$
\widehat{\mathbb{E}}\left[\sup _{s \leq u \leq t}\left|\int_{s}^{u} \eta(r) d B(r)\right|^{p}\right] \leq C_{p} \widehat{\mathbb{E}}\left(\int_{s}^{t}|\eta(r)|^{2} d r\right)^{p / 2}
$$

for all $0 \leq s \leq t \leq T$, where $C_{p}$ is a positive constant depending only on $p$.

Lemma 14 (see [19]). Let $p \geq 1$ and $\eta \in M_{G}^{p}([0, T])$. Then we have

$$
\widehat{\mathbb{E}}\left[\sup _{s \leq u \leq t}\left|\int_{s}^{u} \eta(r) d\langle B\rangle(r)\right|^{p}\right] \leq(t-s)^{p-1} \widehat{\mathbb{E}} \int_{s}^{t}\left[|\eta(r)|^{p}\right] d r
$$

for all $0 \leq s \leq t \leq T$.

\section{Existence and Uniqueness Theorem}

In this section, we prove the existence and uniqueness of solutions to (3) under global Carathéodory conditions. More works for stochastic differential equations driven by $G$ Brownian motion can be found in Bai and Lin [18], Chen and Zhang [20], Gao [19], Lin [21], Lin [22], Ren et al. [23, 24], and the references therein.

Let $\tau>0$ and denote by $C([-\tau, 0] ; \mathbb{R})$ the family of continuous functions $\varphi$ from $[-\tau, 0]$ to $\mathbb{R}$ with the norm

$$
\|\varphi\|=\sup _{-\tau \leq \theta \leq 0}|\varphi(\theta)| .
$$

Given $0 \leq t_{0}<T<\infty$ let

$$
b, h, \sigma:\left[t_{0}, T\right] \times C([-\tau, 0] ; \mathbb{R}) \longrightarrow \mathbb{R}
$$

be three Borel measurable functions satisfying

$$
b(\cdot, x), h(\cdot, x), \sigma(\cdot, x) \in M_{G}^{2}\left(\left[t_{0}, T\right]\right)
$$

for any $x \in C([-\tau, 0] ; \mathbb{R})$. Consider the following SFDE with finite delay of the form

$$
d X(t)=b\left(t, X_{t}\right) d t+h\left(t, X_{t}\right) d\langle B\rangle(t)+\sigma\left(t, X_{t}\right) d B(t),
$$

where $X_{t}=\{X(t+\theta) ;-\tau \leq \theta \leq 0\}$ and $t \in\left[t_{0}, T\right]$ is regarded as a $C([-\tau, 0] ; \mathbb{R})$-valued stochastic process. Then we can impose the initial data:

(i) $X_{t_{0}}=\xi_{t_{0}}=\left\{\xi_{t_{0}}(\theta) ;-\tau \leq \theta \leq 0\right\}$ is an $\mathscr{F}_{t_{0}}$-measurable, $C([-\tau, 0] ; \mathbb{R})$-valued random variable such that $\widehat{\mathbb{E}}\|\xi\|^{2}<\infty$.

Thus we can get the following equivalent form:

$$
\begin{aligned}
X(t)= & \xi_{t_{0}}(0)+\int_{t_{0}}^{t} b\left(s, X_{s}\right) d s+\int_{t_{0}}^{t} h\left(s, X_{s}\right) d\langle B\rangle(s) \\
& +\int_{t_{0}}^{t} \sigma\left(s, X_{s}\right) d B(s)
\end{aligned}
$$


with $t \in\left[t_{0}, T\right]$. In order to give the existence and uniqueness we now propose the following assumptions:

(H1) There exists a function $H(t, u):\left[t_{0}, T\right] \times \mathbb{R}_{+} \rightarrow \mathbb{R}_{+}$, such that

(1a) $|b(t, x)|^{2} \vee|h(t, x)|^{2} \vee|\sigma(t, x)|^{2} \leq H\left(t,|x|^{2}\right)$

(1b) $H(t, u)$ is integrable in $t$ for each fixed $u \in \mathbb{R}_{+}$, and is continuous, concave, nondecreasing in $u$ for each fixed $t \in\left[t_{0}, T\right]$,

(1c) for any constant $K>0$ the deterministic ODE

$$
\frac{d u}{d t}=K H(t, u), \quad t \in\left[t_{0}, T\right],
$$

has a global solution for any initial value.

(H2) There exists a function $\Gamma(t, u):\left[t_{0}, T\right] \times \mathbb{R}_{+} \rightarrow \mathbb{R}_{+}$ such that

$$
\begin{aligned}
& |b(t, x)-b(t, y)|^{2} \\
& \quad \vee|h(t, x)-h(t, y)|^{2} \vee|\sigma(t, x)-\sigma(t, y)|^{2} \\
& \quad \leq \Gamma\left(t,|x-y|^{2}\right)
\end{aligned}
$$$$
\text { for all } x, y \in \mathbb{R} \text { and } t \in\left[t_{0}, T\right] \text {; }
$$

(2b) $\Gamma(t, u)$ is integrable in $t$ for each fixed $u \in \mathbb{R}_{+}$ and is continuous, concave, nondecreasing in $u$ for each fixed $t \geq t_{0}$. Moreover, $\Gamma(t, 0) \equiv 0$, and if there exists a nonnegative continuous function $Z(t), t \in\left[t_{0}, T\right]$ satisfies

$$
Z(t) \leq D \int_{t_{0}}^{t} \Gamma(s, Z(s)) d s, \quad t \in\left[t_{0}, T\right],
$$

where $D>0$ is a positive constant, then $Z(t) \equiv 0$ for all $t \in\left[t_{0}, T\right]$.

Example 15 (see [25]). Let $\Gamma(t, u)=\lambda(t) \alpha(u), t \geq t_{0}, u \geq 0$, where $\lambda(t) \geq 0$ is integrable and $\alpha: \mathbb{R}_{+} \rightarrow \mathbb{R}_{+}$is a continuous, concave, monotone nondecreasing function with $\alpha(0)=0$ such that $\int_{0^{+}}(1 / \alpha(u)) d u=\infty$. Let $u(t)$ be a solution of $d u / d t=\lambda(t) \alpha(u)$. If $u\left(t_{0}\right)=0$, then we get $u(t) \equiv 0$ for all $t \geq t_{0}$. If not, we can suppose without loss of generality that there exists $t_{1}>t_{0}$ such that $u(t)$ is positive for all $t \in\left(t_{0}, t_{1}\right]$. Let $t_{0}<t<t_{1}$. Thus,

$$
\int_{u(t)}^{u\left(t_{1}\right)}\left(\frac{1}{\alpha(u)}\right) d u=\int_{t}^{t_{1}} \lambda(s) d s,
$$

which implies a contradiction letting $t \rightarrow t_{0}$. Therefore, $u(t) \equiv 0$ for all $t \geq t_{0}$.

Our main object of this section is to explain and prove the following theorem.

Theorem 16. Let the assumptions (H1) and (H2) hold. Then, SFDE (37) brings a unique solution $X$ with the above initial data and $X \in \mathbb{L}_{G}^{2}$.
Proof. Define $X_{t_{0}}^{0}=\xi_{t_{0}}, X^{0}(t)=\xi_{t_{0}}(0)$ for $t \in\left[t_{0}, T\right]$, and let $X_{t_{0}}^{n}=\xi_{t_{0}}$ for each $n=1,2, \ldots$. Consider the following Picard iterations:

$$
\begin{aligned}
X^{n}(t)= & \xi_{t_{0}}(0)+\int_{t_{0}}^{t} b\left(s, X_{s}^{n-1}\right) d s+\int_{t_{0}}^{t} h\left(s, X_{s}^{n-1}\right) d\langle B\rangle(s) \\
& +\int_{t_{0}}^{t} \sigma\left(s, X_{s}^{n-1}\right) d B(s)
\end{aligned}
$$

for $n=0,1,2, \ldots$. Then we get

$$
\begin{aligned}
X^{1}(t)= & \xi_{t_{0}}(0)+\int_{t_{0}}^{t} b\left(s, X_{s}^{0}\right) d s+\int_{t_{0}}^{t} h\left(s, X_{s}^{0}\right) d\langle B\rangle(s) \\
& +\int_{t_{0}}^{t} \sigma\left(s, X_{s}^{0}\right) d B(s) .
\end{aligned}
$$

Step 1. We claim that

$$
\begin{gathered}
\widehat{\mathbb{E}}\left|X^{n}(t)\right|^{2} \leq u(t), \\
\widehat{\mathbb{E}}\left|X^{n}(t)-X^{0}(t)\right|^{2} \leq C
\end{gathered}
$$

for all $n=1,2, \ldots$ and all $t \in\left[t_{0}, T\right]$, where $C$ is a positive constant, which points out that there exists a real number $R>$ 0 depending only on $t_{0}$ and $T$ such that

$$
\widehat{\mathbb{E}}\left|X^{n}(t)\right|^{2} \leq R
$$

for all $t \in\left[t_{0}, T\right]$ and all $n=0,1,2, \ldots$, since $u(t)$ is continuous on $\left[t_{0}, T\right]$.

We will use the induction to prove this. It follows from Lemmas 5, 13, and 14 and assumptions (1a), (1b) that there exist three positive constants $k_{1}, k_{2}$, and $k_{3}$ independent of $t$ such that

$$
\begin{aligned}
& \widehat{\mathbb{E}}\left|X^{1}(t)\right|^{2} \\
& \leq 4 \widehat{\mathbb{E}}\left|\xi_{t_{0}}(0)\right|^{2}+4 k_{1} t \widehat{\mathbb{E}} \int_{t_{0}}^{t}\left|b\left(s, X_{s}^{0}\right)\right|^{2} d s \\
& \quad+4 k_{2} t \widehat{\mathbb{E}} \int_{t_{0}}^{t}\left|h\left(s, X_{s}^{0}\right)\right|^{2} d s+4 k_{3} \widehat{\mathbb{E}} \int_{t_{0}}^{t}\left|\sigma\left(s, X_{s}^{0}\right)\right|^{2} d s \\
& \leq 4 \widehat{\mathbb{E}}\|\xi\|^{2}+4\left(k_{1} T+k_{2} T+k_{3}\right) \widehat{\mathbb{E}} \int_{t_{0}}^{t} H\left(s,\left|X_{s}^{0}\right|^{2}\right) d s \\
& \leq 4 \widehat{\mathbb{E}}\|\xi\|^{2}+4\left(k_{1} T+k_{2} T+k_{3}\right) \int_{t_{0}}^{t} H\left(s, \widehat{\mathbb{E}}\left|X_{s}^{0}\right|^{2}\right) d s
\end{aligned}
$$

for all $t \in\left[t_{0}, T\right]$. Notice that $d u / d t=K H(t, u)$ has a global solution with any initial value $\left(t_{0}, u_{0}\right)$. We can take $u_{0} \in \mathbb{R}_{+}$, such that $u_{0}>4 \widehat{\mathbb{E}}\|\xi\|^{2}$, and may assume that $u(t)=u\left(t ; t_{0}, u_{0}\right)$ 
is the solution of the above ODE with the initial value $\left(t_{0}, u_{0}\right)$, which implies that

$$
\begin{aligned}
u(t)- & \widehat{\mathbb{E}}\left|X^{1}(t)\right|^{2} \\
> & 4\left(k_{1} T+k_{2} T+k_{3}\right) \\
& \times \int_{t_{0}}^{t}\left(H(s, u(s))-H\left(s, \hat{\mathbb{E}}\left|X_{s}^{0}\right|^{2}\right)\right) d s \geq 0 .
\end{aligned}
$$

Denote that $p_{0}=\max \left\{u(t) \mid t \in\left[t_{0}, T\right]\right\}$. Then continuity of $u(t)$ on $\left[t_{0}, T\right]$ deduces $p_{0}<\infty$ and

$$
H(s, u(s)) \leq H\left(s, p_{0}\right)
$$

for each $s \in\left[t_{0}, T\right]$. Therefore, there exists a positive constant $C$ such that

$$
\begin{aligned}
& \widehat{\mathbb{E}}\left|X^{1}(t)-X^{0}(t)\right|^{2} \\
& \quad \leq 3\left(k_{1} T+k_{2} T+k_{3}\right) \int_{t_{0}}^{t} H\left(s, p_{0}\right) d s \leq C .
\end{aligned}
$$

We now suppose that the inequalities

$$
\begin{gathered}
\widehat{\mathbb{E}}\left|X^{k}(t)\right|^{2} \leq u(t), \\
\widehat{\mathbb{E}}\left|X^{k}(t)-X^{0}(t)\right|^{2} \leq C
\end{gathered}
$$

hold for all $t \in\left[t_{0}, T\right]$ and $k \geq 1$. Then we have

$$
\begin{aligned}
X^{k+1}(t)=\xi_{t_{0}}(0)+\int_{t_{0}}^{t} b\left(s, X_{s}^{k}\right) d s & \\
& +\int_{t_{0}}^{t} h\left(s, X_{s}^{k}\right) d\langle B\rangle(s)+\int_{t_{0}}^{t} \sigma\left(s, X_{s}^{k}\right) d B(s) \\
\widehat{\mathbb{E}}\left|X^{k+1}(t)\right|^{2} \leq & 4 \widehat{\mathbb{E}}\|\xi\|^{2}+4\left(k_{1} T+k_{2} T+k_{3}\right) \\
& \times \int_{t_{0}}^{t} H\left(s, \widehat{\mathbb{E}}\left|X_{s}^{k}\right|^{2}\right) d s
\end{aligned}
$$

Since $u_{0}>4 \widehat{\mathbb{E}}\|\xi\|^{2}$, we obtain that

$$
\begin{aligned}
u(t)- & \widehat{\mathbb{E}}\left|X^{k+1}(t)\right|^{2} \\
> & 4\left(k_{1} T+k_{2} T+k_{3}\right) \\
& \times \int_{t_{0}}^{t}\left(H(s, u(s))-H\left(s, \widehat{\mathbb{E}}\left|X_{s}^{k}\right|^{2}\right)\right) d s \geq 0 ;
\end{aligned}
$$

then $\widehat{\mathbb{E}}\left|X^{k+1}(t)\right|^{2} \leq u(t)$ for all $t \in\left[t_{0}, T\right]$, and

$$
\begin{aligned}
\widehat{\mathbb{E}}\left|X^{k+1}(t)-X^{0}(t)\right|^{2} \leq & 3\left(k_{1} T+k_{2} T+k_{3}\right) \\
& \times \int_{t_{0}}^{t} H\left(s, p_{0}\right) d s \leq C
\end{aligned}
$$

for all $t \in\left[t_{0}, T\right]$. Thus, we obtain our assertion by induction.
Step 2. We claim that $\left\{X^{n}(t)\right\}$ is a Cauchy sequence in $\mathbb{L}_{G}^{2}$. Thus, $X^{n}(t) \rightarrow X(t)$ in $\mathbb{L}_{G}^{2}$ as $n \rightarrow \infty$. From Lemmas 5,13 , and 14 and assumptions (2a) and (2b), we get, for all $m \geq n \geq 0$,

$$
\begin{aligned}
\widehat{\mathbb{E}}\left(\sup _{t_{0} \leq v \leq t}\left|X^{m}(v)-X^{n}(v)\right|^{2}\right) \\
=\widehat{\mathbb{E}}\left(\sup _{t_{0} \leq v \leq t} \mid \int_{t_{0}}^{v}\left(b\left(s, X_{s}^{m-1}\right)-b\left(s, X_{s}^{n-1}\right)\right) d s\right. \\
+\int_{t_{0}}^{v}\left(h\left(s, X_{s}^{m-1}\right)-h\left(s, X_{s}^{n-1}\right)\right) d\langle B\rangle(s) \\
\left.\quad+\left.\int_{t_{0}}^{v}\left(\sigma\left(s, X_{s}^{m-1}\right)-\sigma\left(s, X_{s}^{n-1}\right)\right) d B(s)\right|^{2}\right) \\
\leq 3 k_{1} T \widehat{\mathbb{E}} \int_{t_{0}}^{t}\left|b\left(v, X_{v}^{m-1}\right)-b\left(v, X_{v}^{n-1}\right)\right|^{2} d v \\
\quad+3 k_{2} T \widehat{\mathbb{E}} \int_{t_{0}}^{T}\left|h\left(v, X_{v}^{m-1}\right)-h\left(v, X_{v}^{n-1}\right)\right|^{2} d v \\
\quad+3 k_{3}^{t} \widehat{\mathbb{E}} \int_{t_{0}}^{t}\left|\sigma\left(v, X_{v}^{m-1}\right)-\sigma\left(v, X_{v}^{n-1}\right)\right|^{2} d v \\
\leq\left(3 k_{1} T+3 k_{2} T+3 k_{3}\right) \\
\left.\quad+3 k_{2} T+3 k_{3}\right) \\
\quad \times \int_{t_{0}}^{t} \Gamma\left(v, \widehat{\mathbb{E}}\left|X_{v}^{m-1}-X_{v}^{n-1}\right|^{2}\right) d v \\
\left.\quad\left(k_{t_{0} \leq s \leq v}\left|X^{m-1}(s)-X^{n-1}(s)\right|^{2}\right)\right) d v .
\end{aligned}
$$

Let $a(t)=\varlimsup_{m, n \rightarrow \infty} \widehat{\mathbb{E}}\left(\sup _{t_{0} \leq v \leq t}\left|X^{m}(v)-X^{n}(v)\right|^{2}\right)$; then we get

$a(t)$

$$
\begin{aligned}
\leq & \left(3 k_{1} T+3 k_{2} T+3 k_{3}\right) \\
& \cdot \int_{t_{0}}^{t}\left(\varlimsup_{m, n \rightarrow \infty} \Gamma\left(v, \widehat{\mathbb{E}}\left(\sup _{t_{0} \leq s \leq v}\left|X^{m-1}(s)-X^{n-1}(s)\right|^{2}\right)\right)\right) d v \\
= & \left(3 k_{1} T+3 k_{2} T+3 k_{3}\right) \\
& \times \int_{t_{0}}^{t} \Gamma\left(v, \varlimsup_{m, n \rightarrow \infty} \widehat{\mathbb{E}}\left(\sup _{t_{0} \leq s \leq v}\left|X^{m-1}(s)-X^{n-1}(s)\right|^{2}\right)\right) d v,
\end{aligned}
$$

which implies that

$$
a(t) \leq\left(3 k_{1} T+3 k_{2} T+3 k_{3}\right) \int_{t_{0}}^{t} \Gamma(s, a(s)) d s .
$$


It follows that $a(t) \equiv 0$ for all $t \in\left[t_{0}, T\right]$. That is,

$$
\varlimsup_{m, n \rightarrow \infty} \widehat{\mathbb{E}}\left(\sup _{t_{0} \leq t \leq T}\left|X^{m}(t)-X^{n}(t)\right|^{2}\right)=0 .
$$

This shows that the sequence $\left\{X^{n}(\cdot)\right\}$ is a Cauchy sequence in $\mathbb{L}_{G}^{2}$, which deduces $X^{n}(\cdot) \rightarrow X(\cdot)$ in $\mathbb{L}_{G}^{2}$ as $n \rightarrow \infty$. Moreover, it is uniformly convergent on $\left[t_{0}, T\right]$, and therefore, $X(t)$ is continuous.

Step 3. We show that the stochastic process $X(t)$ given above is a solution of (37). We have

$$
\begin{aligned}
& \hat{\mathbb{E}}\left|\int_{t_{0}}^{t}\left(b\left(s, X_{s}^{n}\right)-b\left(s, X_{s}\right)\right) d s\right|^{2} \\
& +\widehat{\mathbb{E}}\left|\int_{t_{0}}^{t}\left(h\left(s, X_{s}^{n}\right)-h\left(s, X_{s}\right)\right) d\langle B\rangle(s)\right|^{2} \\
& \quad+\widehat{\mathbb{E}}\left|\int_{t_{0}}^{t}\left(\sigma\left(s, X_{s}^{n}\right)-\sigma\left(s, X_{s}\right)\right) d B(s)\right|^{2} \\
& \leq k_{1} T \widehat{\mathbb{E}} \int_{t_{0}}^{t}\left|b\left(s, X_{s}^{n}\right)-b\left(s, X_{s}\right)\right|^{2} d s \\
& \quad+k_{2} T \widehat{\mathbb{E}} \int_{t_{0}}^{t}\left|h\left(s, X_{s}^{n}\right)-h\left(s, X_{s}\right)\right|^{2} d s \\
& \quad+k_{3} \widehat{\mathbb{E}} \int_{t_{0}}^{t}\left|\sigma\left(s, X_{s}^{n}\right)-\sigma\left(s, X_{s}\right)\right|^{2} d s \\
& \leq\left(k_{1} T+k_{2} T+k_{3}\right) \int_{t_{0}}^{t} \Gamma\left(s, \widehat{\mathbb{E}}\left|X_{s}^{n}-X_{s}\right|^{2}\right) d s
\end{aligned}
$$

for all $t \in\left[t_{0}, T\right]$ and $n \geq 0$. Noting that the sequence $\left\{X^{n}(t)\right\}$ uniformly converges on $\left[t_{0}, T\right]$, we get

$$
\begin{gathered}
\int_{t_{0}}^{t} b\left(s, X_{s}^{n}\right) d s \longrightarrow \int_{t_{0}}^{t} b\left(s, X_{s}\right) d s, \\
\int_{t_{0}}^{t} h\left(s, X_{s}^{n}\right) d\langle B\rangle(s) \longrightarrow \int_{t_{0}}^{t} h\left(s, X_{s}\right) d\langle B\rangle(s), \\
\int_{t_{0}}^{t} \sigma\left(s, X_{s}^{n}\right) d B(s) \longrightarrow \int_{t_{0}}^{t} \sigma\left(s, X_{s}\right) d B(s)
\end{gathered}
$$

in $\mathbb{L}_{G}^{2}$, as $n \rightarrow \infty$, which deduces

$$
\begin{aligned}
X(t)= & \xi_{t_{0}}(0)+\int_{t_{0}}^{t} b\left(s, X_{s}\right) d s+\int_{t_{0}}^{t} h\left(s, X_{s}\right) d\langle B\rangle(s) \\
& +\int_{t_{0}}^{t} \sigma\left(s, X_{s}\right) d B(s) .
\end{aligned}
$$

Thanks to Lemmas 13, 14, and 5 and assumptions (1a), (1b), and (1c), we can also get $X(t) \in \mathbb{L}_{G}^{2}$ for all $t \in\left[t_{0}, T\right]$.

Step 4. Finally, we will show that the uniqueness of the solution. Let $X$ and $Y$ be two solutions existing on $\left[t_{0}, T\right]$ and $X\left(t_{0}\right)=Y\left(t_{0}\right)=\xi_{t_{0}}(0)$. Then, we have

$$
\begin{aligned}
& \widehat{\mathbb{E}}\left(\sup _{t_{0} \leq v \leq t}|X(v)-Y(v)|^{2}\right) \\
& =\widehat{\mathbb{E}}\left[\sup _{t_{0} \leq v \leq t} \mid \int_{t_{0}}^{v}\left(b\left(s, X_{s}\right)-b\left(s, Y_{s}\right)\right) d s\right. \\
& +\int_{t_{0}}^{v}\left(h\left(s, X_{s}\right)-h\left(s, Y_{s}\right)\right) d\langle B\rangle(s) \\
& \left.\quad+\left.\int_{t_{0}}^{v}\left(\sigma\left(s, X_{s}\right)-\sigma\left(s, Y_{s}\right)\right) d B(s)\right|^{2}\right] \\
& \leq\left(3 k_{1} T+3 k_{2} T+3 k_{3}\right) \int_{t_{0}}^{t} \Gamma\left(s, \widehat{\mathbb{E}}\left|X_{s}-Y_{s}\right|^{2}\right) d s \\
& \leq\left(3 k_{1} T+3 k_{2} T+3 k_{3}\right) \\
& \quad \times \int_{t_{0}}^{t} \Gamma\left(s, \widehat{\mathbb{E}}\left(\sup _{t_{0} \leq v \leq s}|X(v)-Y(v)|^{2}\right)\right) d s,
\end{aligned}
$$

which deduces $X(t)=Y(t)$ for all $t \in\left[t_{0}, T\right]$ q.s. This shows that the uniqueness of the solution and the theorem follows.

\section{An Application}

In this section, we consider an application of the above theorem. Suppose that the stock price satisfies the following equation:

$$
\begin{gathered}
d S(t)=b\left(S_{t}\right) S(t) d t+\sigma\left(S_{t}\right) S(t) d B(t) \\
+h\left(S_{t}\right) S(t) d\langle B\rangle(t), \\
S_{0}=\xi=\{\xi(\theta) ;-\tau \leq \theta \leq 0\},
\end{gathered}
$$

where $S_{t}=\{S(t+\theta) ;-\tau \leq \theta \leq 0, t \in[0, T]\}$ is regarded as a $C([-\tau, 0] ; \mathbb{R})$-valued stochastic process. $\{\langle B\rangle(t), t \geq 0\}$ is the quadratic variation process of $G$-Brownian motion $\{B(t), t \geq$ $0\}$.

In the rest of this section, we want to consider a special case of the above equation. We let the functions

$$
b, \sigma, h: C([-\tau, 0] ; \mathbb{R}) \longrightarrow \mathbb{R}
$$

satisfy the following conditions:

(1) there exists a $\varepsilon_{1}>0$ such that $|\sigma(x)|>\varepsilon_{1}$;

(2) $|b(x)|>r$, where $r>0$ denotes the rate of return on a riskless asset, which is compounded continuously;

(3) there exists a $M>0$, such that $|b(x)| \vee|\sigma(x)| \vee|h(x)| \leq$ M. 
Corollary 17. Under the above assumption, the above SDDE brings a unique solution and

$$
\begin{aligned}
S(t)=\xi(0) \exp \left\{\int_{0}^{t} b\left(S_{v}\right) d v+\int_{0}^{t} \sigma\left(S_{v}\right) d B(v)\right. \\
\left.+\int_{0}^{t}\left[\left(h\left(S_{v}\right)\right)^{2}-\frac{1}{2}\left(\sigma\left(S_{v}\right)\right)^{2}\right] d\langle B\rangle(v)\right\} .
\end{aligned}
$$

Furthermore, if $\xi(0) \geq 0(\xi(0)>0)$ q.s., then $S(t) \geq 0(S(t)>$ 0 ) for all $t \geq 0$ q.s.

Proof. Let $\theta_{0} \in[-\tau, 0]$. Consider the case $t \in\left[0,-\theta_{0}\right]$, and in this situation, we have $\theta_{0} \leq t+\theta_{0} \leq 0$ and

$$
S_{t}=S\left(t+\theta_{0}\right)=\xi\left(t+\theta_{0}\right),
$$

which deduces

$$
\begin{gathered}
d S(t)=b\left(\xi\left(t+\theta_{0}\right)\right) S(t) d t+\sigma\left(\xi\left(t+\theta_{0}\right)\right) S(t) d B(t) \\
+h\left(\xi\left(t+\theta_{0}\right)\right) S(t) d\langle B\rangle(t), \\
S(0)=\xi(0) .
\end{gathered}
$$

It follows from Theorem 16 and Itô's formula that the unique solution of (67)

$S(t)$

$$
\begin{aligned}
=\xi(0) \exp & \left\{\int_{0}^{t} b\left(\xi\left(v+\theta_{0}\right)\right) d v+\int_{0}^{t} \sigma\left(\xi\left(v+\theta_{0}\right)\right) d B(v)\right. \\
& +\int_{0}^{t}\left[\left(h\left(\xi\left(v+\theta_{0}\right)\right)\right)^{2}-\frac{1}{2}\left(\sigma\left(\xi\left(v+\theta_{0}\right)\right)\right)^{2}\right] \\
& \times d\langle B\rangle(v)\}
\end{aligned}
$$

for all $t \in\left[0,-\theta_{0}\right]$. This implies that $S(t)>0$ for all $t \in$ $\left[0,-\theta_{0}\right]$, when $\xi(0)>0$ q.s. By a similar argument, it follows that $S(t)>0$ for all $t \in\left[-\theta_{0},-2 \theta_{0}\right]$ q.s. Therefore $S(t)>0$ for all $t \geq 0$ q.s. By induction, we can also get the solution to (67) as follows:

$S(t)$

$$
\begin{aligned}
=\xi(0) \exp & \left\{\int_{0}^{t} b\left(S\left(v+\theta_{0}\right)\right) d v+\int_{0}^{t} \sigma\left(S\left(v+\theta_{0}\right)\right) d B(v)\right. \\
& +\int_{0}^{t}\left[\left(h\left(S\left(v+\theta_{0}\right)\right)\right)^{2}-\frac{1}{2}\left(\sigma\left(S\left(v+\theta_{0}\right)\right)\right)^{2}\right] \\
& \times d\langle B\rangle(v)\}
\end{aligned}
$$

for $t \in[0, T]$. Since that equality is true for all $-\tau \leq \theta_{0} \leq 0$, we get our result.
We denote the wealth process by $V(t)$ and units of the stock that we hold by $\alpha(t)$. Thanks to the assumption of selffinancing, we can get

$$
\begin{aligned}
d V(t)= & r(V(t)-\alpha(t) S(t)) d t+\alpha(t) d S(t) \\
= & r(V(t)-\alpha(t) S(t)) d t \\
& +\alpha(t)\left[b\left(S_{t}\right) S(t) d t+\sigma\left(S_{t}\right) S(t) d B(t)\right. \\
& \left.+h\left(S_{t}\right) S(t) d\langle B\rangle(t)\right]
\end{aligned}
$$

for all $t \in[0, T]$. Let

$$
H(t, \omega)=\frac{\left(\left(r-b\left(S_{t}\right)\right) / \sigma_{0}^{2}\right)-h\left(S_{t}\right)}{\sigma\left(S_{t}\right)} .
$$

Then there exists a $\varepsilon_{0}>0$ such that

$$
\widehat{\mathbb{E}} \exp \left[\left(\frac{1}{2}+\varepsilon_{0}\right) \int_{0}^{T} H^{2}(t, \omega) d\langle B\rangle(t)\right]<\infty,
$$

which shows that

$$
\widetilde{B}(t)=B(t)-\int_{0}^{t} H(s, \omega) d\langle B\rangle(s)
$$

is $G$-Brownian motion under some $G$-expectation $\widetilde{\mathbb{E}}$ by $G$ Girsanov theorem. Let now $\Gamma(t)=e^{-r t}$; then

$$
\begin{gathered}
d \Gamma(t)=-r \Gamma(t) d t \\
d \Gamma(t) V(t)=\Gamma(t) d V(t)+V(t) d \Gamma(t)+d \Gamma(t) d V(t) \\
=-r \Gamma(t) V(t) d t+\Gamma(t) d V(t) \\
=\alpha(t) \Gamma(t) S(t)\left[\left(b\left(S_{t}\right)-r\right) d t\right. \\
+\sigma\left(S_{t}\right) d B(t) \\
\left.+h\left(S_{t}\right) d\langle B\rangle(t)\right] \\
=\alpha(t) \Gamma(t) S(t)\left[\begin{array}{l}
\left(b\left(S_{t}\right)-r\right) d t \\
+\sigma\left(S_{t}\right) d \widetilde{B}(t)
\end{array}\right. \\
\left.+\frac{r-b\left(S_{t}\right)}{\sigma_{0}^{2}} d\langle B\rangle(t)\right] \\
+\alpha(t) \Gamma(t) S(t) \sigma\left(S_{t}\right) d \widetilde{B}(t),
\end{gathered}
$$

where $D(t)=\left(b\left(S_{t}\right)-r\right) / \sigma_{0}^{2}$; we can get that the discounted wealth process is $G$-martingale under some $G$-expectation. Thus, we can obtain that the price of European call option $C(0)$ is

$$
C(0) \leq \widetilde{\mathbb{E}}[V(T) \Gamma(T)] .
$$




\section{Acknowledgments}

This work is supported by the Project sponsored by NSFC (no. 11171062), Innovation Program of Shanghai Municipal Education Commission (no. 12ZZ063), and the Project sponsored by CUSF-DH-D-2013038.

\section{References}

[1] S. Peng, "G-expectation, G-Brownian motion and related stochastic calculus of Itô type," in Stochastic Analysis and Applications, vol. 2 of Abel Symposia, pp. 541-567, Springer, Berlin, Germany, 2007.

[2] S. Peng, "Multi-dimensional G-Brownian motion and related stochastic calculus under G-expectation," Stochastic Processes and Their Applications, vol. 118, no. 12, pp. 2223-2253, 2008.

[3] S. Peng, " $G$-Brownian motion and dynamic risk measure under volatility uncertainty," http://arxiv.org/abs/0711.2834.

[4] S. Peng, "Nonlinear expections and stochastic calculus under uncertainty," http://arxiv.org/abs/1002.4546.

[5] F. Black and M. Scholes, "The pricing of options and corporate liabilities," Journal of Political Economy, vol. 81, pp. 637-659, 1973.

[6] R. C. Merton, “Theory of rational option pricing," The Bell Journal of Economics and Management Science, vol. 4, no. 1, pp. 141-183, 1973.

[7] M. Arriojas, Y. Hu, S.-E. Mohammed, and G. Pap, "A delayed black and scholes formula," Stochastic Analysis and Applications, vol. 25, no. 2, pp. 471-492, 2007.

[8] L. Denis, M. Hu, and S. Peng, "Function spaces and capacity related to a sublinear expectation: application to $G$-Brownian motion paths," Potential Analysis, vol. 34, no. 2, pp. 139-161, 2011.

[9] M.-S. Hu and S.-G. Peng, "On representation theorem of Gexpectations and paths of G-Brownian motion," Acta Mathematica Sinica, vol. 25, no. 3, pp. 539-546, 2009.

[10] M. Hu and X. Li, "Independence under the G-expectation framework," Journal of Theoretical Probability, 2012.

[11] Q. Lin, "Local time and tanaka formula for the G-Brownian motion," Journal of Mathematical Analysis and Applications, vol. 398, no. 1, pp. 315-334, 2013.

[12] S. Peng, Y. Song, and J. Zhang, "A complete representation theorem for G-martingales," http://arxiv.org/abs/1201.2629.

[13] Y. Song, "Some properties on $G$-evaluation and its applications to G-martingale decomposition," Science China Mathematics, vol. 54, no. 2, pp. 287-300, 2011.

[14] J. Xu, H. Shang, and B. Zhang, "A Girsanov type theorem under G-framework," Stochastic Analysis and Applications, vol. 29, no. 3, pp. 386-406, 2011.

[15] L. Yan, B. Gao, and Q. Zhang, "The fractional derivatives for GBrownian local time".

[16] L. Yan, X. Sun, and B. Gao, "Integral with respect to the GBrownian local time," http://arxiv.org/abs/1212.6353.

[17] X. Li and S. Peng, "Stopping times and related Itô's calculus with G-Brownian motion," Stochastic Processes and Their Applications, vol. 121, no. 7, pp. 1492-1508, 2011.

[18] X. Bai and Y. Lin, "On the existence and uniqueness of solutions to stochastic differential equations driven by $G$-Brownian motion with integral-lipschitz coefficients," http://arxiv.org/ abs/1002.1046v3.
[19] F. Gao, "Pathwise properties and homeomorphic flows for stochastic differential equations driven by G-Brownian motion," Stochastic Processes and Their Applications, vol. 119, no. 10, pp. 3356-3382, 2009.

[20] Z. Chen and D. Zhang, "Exponential stability for stochastic differential equation driven by $G$-Brownian motion," Applied Mathematics Letters, vol. 25, no. 11, pp. 1906-1910, 2012.

[21] Q. Lin, "Properties of solutions of stochastic differential equations driven by the G-Brownian motion," http://arxiv.org/abs/ 1010.3158 .

[22] Y. Lin, "Stochastic differential equations driven by G-Brownian motion with reflecting boundary conditions," Electronic Journal of Probability, vol. 18, article 9, pp. 1-23, 2013.

[23] Y. Ren, Q. Bi, and R. Sakthivel, "Stochastic functional differential equations with infinite delay driven by $G$-Brownian motion," Mathematical Methods in the Applied Sciences, vol. 36, no. 13, pp. 1746-1759, 2013.

[24] Y. Ren and L. Hu, "A note on the stochastic differential equations driven by G-Brownian motion," Statistics \& Probability Letters, vol. 81, no. 5, pp. 580-585, 2011.

[25] T. Taniguchi, "Successive approximations to solutions of stochastic differential equations," Journal of Differential Equations, vol. 96, no. 1, pp. 152-169, 1992. 


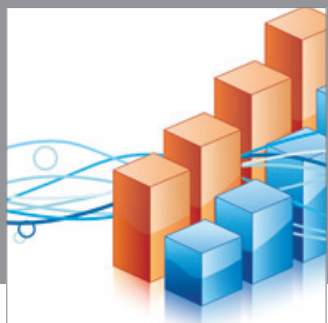

Advances in

Operations Research

mansans

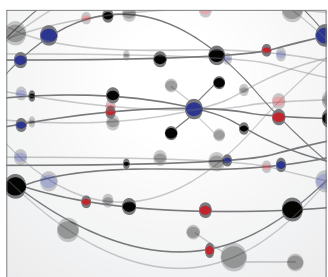

The Scientific World Journal
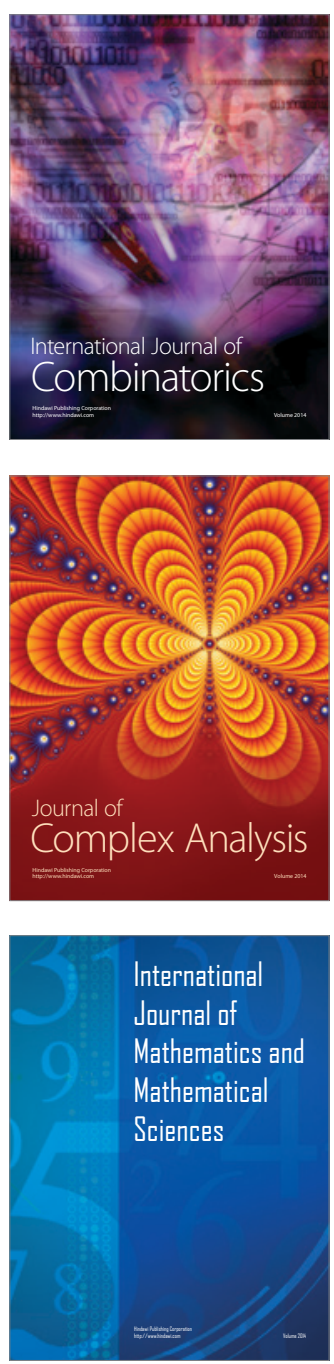
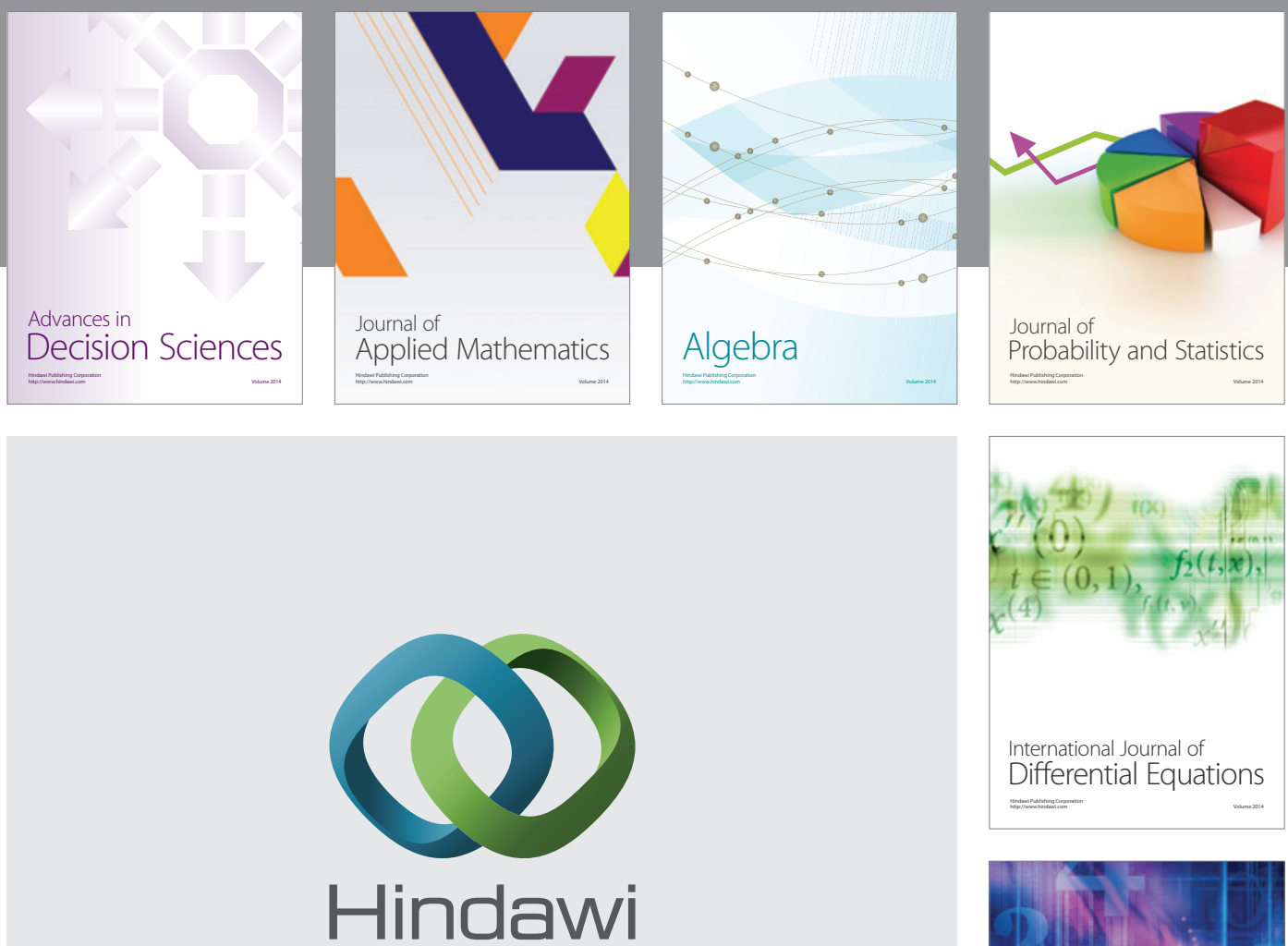

Submit your manuscripts at http://www.hindawi.com
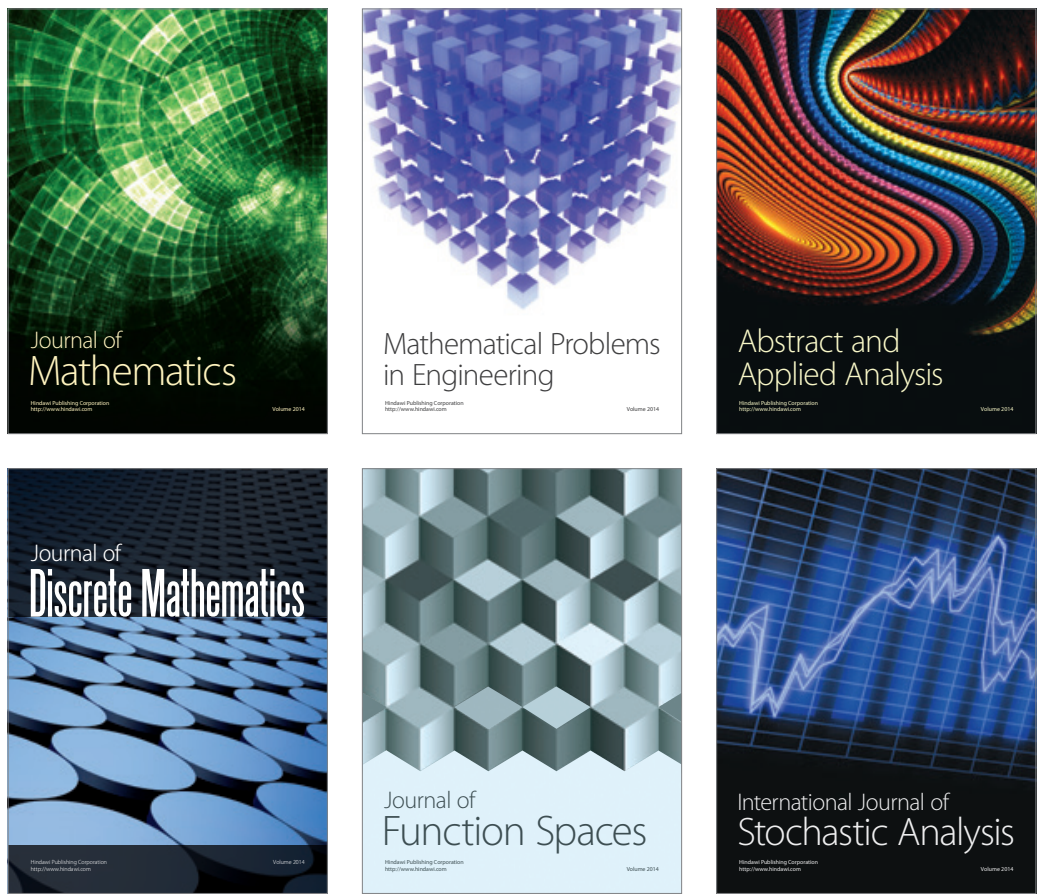

Journal of

Function Spaces

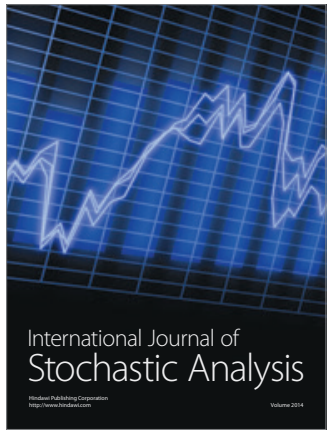

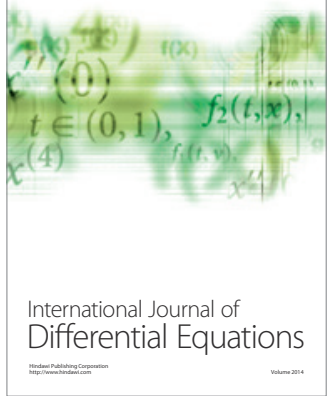
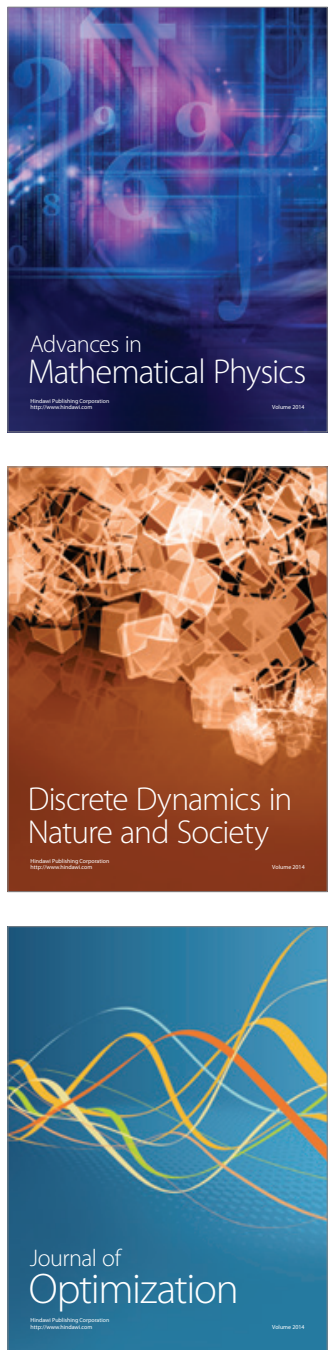